Pacific

Journal of

Mathematics

THE MONGE-AMPÈRE EQUATION WITH INFINITE BOUNDARY VALUE

Bo Guan and Huai-Yu Jian 


\title{
THE MONGE-AMPĖRE EQUATION WITH INFINITE BOUNDARY VALUE
}

\author{
Bo Guan and Huai-Yu Jian
}

\begin{abstract}
This article concerns the Monge-Ampère equations with infinite boundary value in convex domains in Euclidean space. We were able to characterize the growth rate conditions, which are nearly optimal, for the existence/nonexistence of solutions to the problem.
\end{abstract}

\section{Introduction}

Let $\Omega$ be a domain in $\mathbb{R}^{n}$ and $\psi$ a positive function defined on $\Omega \times \mathbb{R} \times \mathbb{R}^{n}$. In this paper we study the Dirichlet problem for the Monge-Ampère equation

$$
\operatorname{det} D^{2} u=\psi(x, u, D u)>0 \text { in } \Omega,
$$

with the infinite boundary value condition

$$
u=+\infty \text { on } \partial \Omega \text {. }
$$

We will look for strictly convex solutions in $C^{\infty}(\Omega)$; it is necessary to assume the underlying domain $\Omega$ to be convex for such solutions to exist.

This problem was first considered by Cheng and Yau $([\mathbf{5}],[\mathbf{6}])$ for $\psi(x, u)=$ $e^{K u} f(x)$ in bounded convex domains and for $\psi(u)=e^{2 u}$ in unbounded domains. More recently, Matero [11] treated the case $\psi=\psi(x, u)$ for bounded strictly convex domains, generalizing a result of Keller [8] and Osserman [12] for the Laplace operator; his results were further extended by Salani [13] to some Hessian equations. (See also [9], where problem (1.1)-(1.2) was studied for $\psi(x, u)=e^{u} f(x)$ and $\psi(x, u)=u^{p} f(x)$.) For the complex Monge-Ampère equation with $\psi(z, u)=e^{K u} f(z)$ the corresponding problem was also treated in [5] in connection with the problem of finding complete Kähler-Einstein metrics on pseudoconvex domains. In this article we will consider more general cases, including allowing domains that are unbounded and not strictly convex when $\psi=\psi(x, u)$. Our main results are stated as follows:

Theorem 1.1. Let $\Omega$ be a bounded strictly convex domain. Suppose that $\psi \in C^{\infty}\left(\bar{\Omega} \times \mathbb{R} \times \mathbb{R}^{n}\right)$ satisfies $\psi>0$,

$$
M\left(z^{+}\right)^{p} \leq \psi(x, z, \mathbf{p}), \quad \forall(x, z, \mathbf{p}) \in \Omega \times \mathbb{R} \times \mathbb{R}^{n},
$$


where $p>n, M>0, z^{+}=\max \{z, 0\}$, and finally

$$
\psi(x, z, \mathbf{p}) \leq \Psi(z)\left(1+|\mathbf{p}|^{n}\right), \quad \forall(x, z, \mathbf{p}) \in \Omega \times \mathbb{R} \times \mathbb{R}^{n},
$$

where $\Psi$ is a smooth positive function and

$$
\sup _{z \leq 0} e^{-\varepsilon z} \Psi(z)<+\infty
$$

for some $\varepsilon>0$. Then there exists a strictly convex solution $u \in C^{\infty}(\Omega)$ to (1.1)-(1.2). Moreover, there exist functions $\underline{h}, \bar{h} \in C\left(\mathbb{R}^{+}\right)$with $\underline{h}(r), \bar{h}(r) \rightarrow$ $\infty$ as $r \rightarrow 0$, such that

$$
\underline{h}(d(x)) \leq u(x) \leq \bar{h}(d(x)), \quad \forall x \in \Omega,
$$

where $d$ is the distance function to $\partial \Omega$.

When $\psi$ does not depend on $D u$, Theorem 1.1 holds under weaker conditions. In particular, $\Omega$ need not be bounded or strictly convex:

Theorem 1.2. Let $\Omega \subset \mathbb{R}^{n}$ be a convex domain not containing a straight line. Suppose $\psi \in C^{\infty}(\bar{\Omega} \times \mathbb{R})$ satisfies $\psi>0$, as well as (1.3) for some $p>n$ and also

$$
\sup _{x \in \Omega, z \leq 0} e^{-\varepsilon z} \psi(x, z)<+\infty
$$

for some $\varepsilon>0$. Then (1.1)-(1.2) has a strictly convex solution $u \in C^{\infty}(\Omega)$ that satisfies (1.6). In addition, when $\Omega$ is bounded, assumption (1.7) can be weakened to allow $\varepsilon=0$.

Remark 1.3. Suppose $\psi_{z} \geq 0$ and there exists a convex supersolution $\bar{u} \in$ $C^{2}(\Omega)$ satisfying

$$
\begin{aligned}
\operatorname{det} D^{2} \bar{u} & \leq \psi(x, \bar{u}, D \bar{u}) \text { in } \Omega, \\
\bar{u} & =\infty \text { on } \partial \Omega .
\end{aligned}
$$

Theorems 1.1 and 1.2 then remain valid, with $\bar{u}(x)$ in place of the function $\bar{h}(d(x))$ in (1.6), without assumption (1.3).

The following nonexistence results complement Theorems 1.1 and 1.2 and indicate that the growth conditions in Theorems 1.1 and 1.2 are nearly optimal.

Theorem 1.4. Let $\Omega$ be a bounded convex domain in $\mathbb{R}^{n}$. If

$$
0 \leq \psi(x, z, \mathbf{p}) \leq M\left(1+\left(z^{+}\right)^{p}\right)\left(1+|\mathbf{p}|^{q}\right), \quad \forall(x, z, \mathbf{p}) \in \Omega \times \mathbb{R} \times \mathbb{R}^{n},
$$

for some $p, q \geq 0, p+q \leq n$, there exists no convex solution to (1.1)-(1.2).

Theorem 1.5. Let $\Omega$ be a convex domain in $\mathbb{R}^{n}$. If

$$
\psi(x, z, \mathbf{p}) \geq M\left(1+|\mathbf{p}|^{n}\right)^{\alpha}, \quad \forall(x, z, \mathbf{p}) \in \Omega \times \mathbb{R} \times \mathbb{R}^{n},
$$

where $\alpha>1$ and $M>0$, and $\Omega$ contains a ball of radius $a>(M(\alpha-1))^{-1 / n}$, there exists no convex solution to (1.1)-(1.2). 
Note that $\Omega$ is not assumed to be bounded in Theorem 1.5.

Theorem 1.6. Assume $\Omega$ is an unbounded convex domain that contains a straight line. If $\psi>0$ satisfies (1.3), where $p>n$, there is no convex solution to (1.1)-(1.2) in $C^{2}(\Omega)$.

The article is organized as follows: we start with some comparison principle and uniqueness results in Section 2. In Section 3 we construct some radially symmetric functions that will be used as barriers in proving our theorems. Section 3 also contains the proofs of Theorems 1.4-1.6, while Theorems 1.1 and 1.2 are proved in Sections 4 and 5.

\section{The comparison principle and uniqueness}

Throughout this section $\Omega \subset \mathbb{R}^{n}$ is assumed to be a bounded convex domain and $u, v \in C^{2}(\Omega)$ are convex functions satisfying $\operatorname{det} D^{2} u \geq \psi(x, u, D u)$ and $\operatorname{det} D^{2} v \leq \phi(x, v, D v)$ in $\Omega$, where $\psi, \phi \in C^{2}\left(\Omega \times \mathbb{R} \times \mathbb{R}^{n}\right)$ and

$$
\psi(x, z, \mathbf{p}) \geq \phi(x, z, \mathbf{p}) \geq 0, \quad \forall(x, z, \mathbf{p}) \in \Omega \times \mathbb{R} \times \mathbb{R}^{n} .
$$

For later reference we recall the following comparison principle, which will be used repeatedly:

Lemma 2.1. Assume $u, v \in C(\bar{\Omega})$ and $u \leq v$ on $\partial \Omega$. If either $\psi_{z}(x, z, \mathbf{p})>$ 0 or $\phi_{z}(x, z, \mathbf{p})>0$ for any $(x, z, \mathbf{p}) \in \Omega \times \mathbb{R} \times \mathbb{R}^{n}$, then $u \leq v$ in $\Omega$.

Proof. Assume that

$$
u(y)-v(y)=\max _{\Omega}(u-v)>0
$$

for some $y \in \Omega$. Then $\operatorname{det} D^{2} u(y) \leq \operatorname{det} D^{2} v(y)$, as the Hessian $D^{2}(v-u)$ is positive semidefinite at $y$. On the other hand, we have

$$
\operatorname{det} D^{2} v(y) \leq \phi(y, v(y), D v(y))<\psi(y, u(y), D u(y)) \leq \operatorname{det} D^{2} u(y),
$$

since $u(y)>v(y)$ and $D u(y)=D v(y)$. This contradiction shows $u \leq v$ in $\Omega$.

We have the following comparison principle and uniqueness for solutions of problem (1.1)-(1.2):

Theorem 2.2. Assume $u=+\infty, v=+\infty$ on $\partial \Omega$ and $v$ is strictly convex in $\Omega$. Suppose $\Omega$ contains the origin in $\mathbb{R}^{n}$ and $\psi$ satisfies

$$
x \cdot D_{x} \psi(x, z, \mathbf{p}) \leq 0, \mathbf{p} \cdot D_{\mathbf{p}} \psi(x, z, \mathbf{p}) \geq 0, \quad \forall(x, z, \mathbf{p}) \in \Omega \times \mathbb{R} \times \mathbb{R}^{n} .
$$

If, in addition, either

$$
\psi\left(x, \lambda z^{+}, \mathbf{p}\right) \geq \lambda^{p} \psi(x, z, \mathbf{p}), \quad \forall \lambda \geq 1,(x, z, \mathbf{p}) \in \Omega \times \mathbb{R} \times \mathbb{R}^{n},
$$


where $p>n$, or there exists $\varepsilon>0$ such that

$$
\psi_{z}(x, z, \mathbf{p}) \geq \varepsilon \psi(x, z, \mathbf{p}), \quad \forall(x, z, \mathbf{p}) \in \Omega \times \mathbb{R} \times \mathbb{R}^{n},
$$

then $u \leq v$ in $\Omega$. In particular, problem (1.1)-(1.2) admits at most one strictly convex solution in $C^{2}(\Omega)$.

Remark 2.3. Assumption (2.4) implies that $\psi=0$ where $z \leq 0$. Thus any strictly convex solution of (1.1)-(1.2) must be positive when (2.4) is satisfied. Note also that $\psi_{z}>0$ wherever $\psi>0$ if either (2.4) or (2.5) holds.

Remark 2.4. If $\psi_{z}(x, z, \mathbf{p}) \geq 0$, then $\left(z^{+}\right)^{p} \psi(x, z, \mathbf{p})$ satisfies $(2.4)$ and $e^{\varepsilon z} \psi(x, z, \mathbf{p})$ satisfies $(2.5)$.

Proof of Theorem 2.2. Let $u \in C^{2}(\Omega)$ be a convex solution of (1.1)-(1.2). Consider for $0<\lambda \leq 1$,

$$
u_{\lambda}(x):=\lambda^{\alpha} u(\lambda x)-a, \quad x \in \Omega_{\lambda},
$$

where $\Omega_{\lambda}=\left\{x \in \mathbb{R}^{n}: \lambda x \in \Omega\right\}$ and

$$
\begin{cases}a=0, \alpha=2 n /(p-n), & \text { if (2.4) holds, } \\ \alpha=0, a=\varepsilon^{-1} \lambda^{-(2+\alpha) n}, & \text { if (2.5) holds. }\end{cases}
$$

We calculate

$$
\begin{aligned}
\operatorname{det} D^{2} u_{\lambda}(x) & =\lambda^{(2+\alpha) n} \operatorname{det} D^{2} u(\lambda x) \\
& =\lambda^{(2+\alpha) n} \psi(\lambda x, u(\lambda x), D u(\lambda x)) \\
& =\lambda^{(2+\alpha) n} \psi\left(\lambda x, \lambda^{-\alpha}\left(u_{\lambda}(x)+a\right), \lambda^{-(1+\alpha)} D u_{\lambda}(x)\right) \\
& \geq \lambda^{(2+\alpha) n} \psi\left(x, \lambda^{-\alpha}\left(u_{\lambda}(x)+a\right), D u_{\lambda}(x)\right) \\
& \geq \psi\left(x, u_{\lambda}(x), D u_{\lambda}(x)\right)
\end{aligned}
$$

by assumption (2.3), when either (2.4) or (2.5) holds.

Now note that $\bar{\Omega} \subset \Omega_{\lambda}$ and $v-u_{\lambda}=+\infty$ on $\partial \Omega$ for all $0<\lambda<1$. We claim that $v \geq u_{\lambda}$ on $\Omega$ for all $0<\lambda<1$. Indeed, assume that

$$
u_{\lambda}(y)-v(y)=\max _{\Omega}\left(u_{\lambda}-v\right)>0
$$

for some $y \in \Omega$. Then

$$
\begin{aligned}
\psi\left(y, u_{\lambda}(y), D u_{\lambda}(y)\right) & \geq \psi(y, v(y), D v(y)) \\
& \geq \phi(y, v(y), D v(y)) \geq \operatorname{det} D^{2} v>0 .
\end{aligned}
$$

It follows that $\psi_{z}\left(y, u_{\lambda}(y), D u_{\lambda}\right)(y)>0$; see Remark 2.3. Consequently, we obtain a contradiction as in the proof of Lemma 2.1. This proves our claim, that is, $v \geq u_{\lambda}$ on $\Omega$ for all $0<\lambda<1$. Letting $\lambda \rightarrow 1$ we obtain $v \geq u$. 


\section{Barriers}

The main purpose of this section is to construct some radially symmetric strictly convex functions that will be used as barriers in proving our main results. Using these barriers we present proofs of Theorems 1.4-1.6 at the end of this section.

Let $u(x)=u(|x|)$ be a radially symmetric function. A straightforward calculation shows that

$$
\operatorname{det} D^{2} u=\left(\frac{u^{\prime}}{r}\right)^{n-1} u^{\prime \prime}, \quad r=|x| .
$$

Thus Equation (1.1) takes the form

$$
\left(u^{\prime}\right)^{n-1} u^{\prime \prime}=r^{n-1} \psi\left(x, u, u^{\prime}\right)
$$

for radially symmetric functions.

Lemma 3.1. Let $\eta \in C^{1}(\mathbb{R})$ satisfy $\eta(z)>0, \eta^{\prime}(z) \geq 0$ for all $z \in \mathbb{R}$. Then, for any $a>0$, there exists a strictly convex radially symmetric function $v \in C^{2}\left(B_{a}(0)\right)$ satisfying

$$
\begin{aligned}
\operatorname{det} D^{2} v & \geq e^{v} \eta(v)\left(1+|D v|^{n}\right) \text { in } B_{a}(0), \\
v & =+\infty \text { on } \partial B_{a}(0) .
\end{aligned}
$$

Proof. Consider the initial value problem

$$
\begin{aligned}
\varphi^{\prime} & =\left(\exp \left(r^{n} e^{\varphi} \eta(\varphi)\right)-1\right)^{1 / n}, r>0 \\
\varphi(0) & =0 .
\end{aligned}
$$

Let $[0, R)$ be the maximal interval on which the solution to (3.4) exists. We claim that $R$ is finite. Indeed, by (3.4) we have

$$
\varphi^{\prime}(r) \geq r\left(e^{\varphi(r)} \eta(\varphi(r))\right)^{1 / n} \geq r\left(e^{\varphi(r)} \eta(0)\right)^{1 / n}, \quad 0<r<R,
$$

since $\eta^{\prime} \geq 0, \varphi^{\prime} \geq 0$ and $\varphi(0)=0$. It follows that

$$
\begin{aligned}
n \geq n\left(1-e^{-\varphi(\rho) / n}\right) \geq \int_{0}^{\rho} \varphi^{\prime}(r) e^{-\varphi(r) / n} d r & \geq(\eta(0))^{1 / n} \int_{0}^{\rho} r d r \\
& =\frac{1}{2}(\eta(0))^{1 / n} \rho^{2}
\end{aligned}
$$

for any $\rho<R$. This proves that $R<\infty$. Moreover, by the theory of ordinary differential equations we see that $\varphi \in C^{2}[0, R)$ and $\varphi(R)=+\infty$ as $\varphi$ is strictly increasing. Rewriting (3.4) in the form

$$
\log \left(1+\left(\varphi^{\prime}\right)^{n}\right)=r^{n} e^{\varphi} \eta(\varphi)
$$

we obtain by differentiation

$$
\frac{n\left(\varphi^{\prime}\right)^{n-1} \varphi^{\prime \prime}}{1+\left(\varphi^{\prime}\right)^{n}}=\left(r^{n} e^{\varphi} \eta(\varphi)\right)^{\prime} \geq n r^{n-1} e^{\varphi} \eta(\varphi), \quad 0<r<R .
$$

In particular, $\varphi^{\prime \prime}(r)>0$ for $0<r<R$. 
For given $a>0$, let $v$ be defined by

$$
v(x):=\varphi(\lambda|x|)-2 n(-\log \lambda)^{+}, \quad x \in B_{a}(0),
$$

where $\lambda=R / a$. Note that $\varphi^{\prime}(0)=0$. We see that $v$ lies in $C^{2}\left(B_{a}(0)\right)$ and is strictly convex since $\varphi \in C^{2}[0, R)$ and $\varphi^{\prime \prime}>0$. By (3.1) and (3.5) we obtain in $B_{a}(0)$

$$
\begin{aligned}
\operatorname{det} D^{2} v(x) & =\lambda^{2 n} \frac{\left(\varphi^{\prime}(\lambda|x|)\right)^{n-1} \varphi^{\prime \prime}(\lambda|x|)}{(\lambda|x|)^{n-1}} \\
& \geq \lambda^{2 n} e^{\varphi(\lambda|x|)} \eta(\varphi(\lambda|x|))\left(1+\left(\varphi^{\prime}(\lambda|x|)\right)^{n}\right) \\
& \geq \lambda^{2 n} e^{v(x)+2 n(-\log \lambda)^{+}} \eta\left(v(x)+2 n(-\log \lambda)^{+}\right)\left(1+\lambda^{-n}|D v(x)|^{n}\right) \\
& \geq e^{v(x)} \eta(v(x))\left(1+|D v(x)|^{n}\right) .
\end{aligned}
$$

In the last inequality we used the fact that $\eta$ is nondecreasing. The proof of Lemma 3.1 is complete.

Remark 3.2. In the sequel we will denote the function $v \in C^{2}\left(B_{a}(0)\right)$ in Lemma 3.1 by $v^{a, \eta}$. We will also write $v^{a, \eta}(x)=v^{a, \eta}(|x|)$, since it is radially symmetric.

By Lemma 2.1 we have:

Lemma 3.3. Let $u \in C^{2}(\Omega)$ be a strictly convex solution of (1.1)-(1.2), where $\Omega$ is a bounded convex domain contained in a ball $B_{a}\left(x_{0}\right)$. Suppose

$$
\psi(x, z, \mathbf{p}) \leq e^{z} \eta(z)\left(1+|\mathbf{p}|^{n}\right), \quad \forall(x, z, \mathbf{p}) \in \bar{\Omega} \times \mathbb{R} \times \mathbb{R}^{n},
$$

where $\eta \in C^{1}(\mathbb{R})$ satisfies $\eta(z)>0$ and $\eta^{\prime}(z) \geq 0$. Then $u(x) \geq v^{a, \eta}\left(x-x_{0}\right)$ for all $x \in \Omega$.

Proof. We may assume $x_{0}=0$. For any $r>a$, note that $u-v^{r, \eta}=+\infty$ on $\partial \Omega$. Lemma 2.1 implies that $u \geq v^{r, \eta}$ in $\Omega$. Letting $r \rightarrow a$ we obtain $u \geq v^{a, \eta}$.

We next construct a function on $B_{a}(0)$ that will serve as an upper barrier when $\psi$ satisfies (1.3) with $p>n$. A straightforward calculation shows that when $p>n$ the function

$$
w(x):=\left(1-|x|^{2}\right)^{(n+1) /(n-p)}
$$

is strictly convex and satisfies the inequality

$$
\operatorname{det} D^{2} w \leq C(n, p) w^{p} \text { in } B_{1}(0),
$$

where $C(n, p)=p[2(n+1) /(p-n)]^{n+1}$. By rescaling, we have:

Lemma 3.4. Let $a, M>0$ and $p>n$ and define $w^{a, M} \in C^{\infty}\left(B_{a}(0)\right)$ by

$$
w^{a, M}(x):=\lambda w\left(\frac{x}{a}\right), \quad x \in B_{a}(0),
$$


where

$$
\lambda=\left(\frac{C(n, p)}{a^{2 n} M}\right)^{1 /(p-n)} .
$$

Then

$$
\operatorname{det} D^{2} w^{a, M} \leq M\left(w^{a, M}\right)^{p} \text { in } B_{a}(0) .
$$

Proof. One calculates directly

$$
\begin{aligned}
\operatorname{det} D^{2} w^{a, M}(x) & =\frac{\lambda^{n}}{a^{2 n}} \operatorname{det} D^{2} w\left(\frac{x}{a}\right) \\
& \leq \frac{\lambda^{n}}{a^{2 n}} C(n, p)\left(w\left(\frac{x}{a}\right)\right)^{p}=M\left(w^{a, M}(x)\right)^{p} .
\end{aligned}
$$

This completes the proof.

From Lemmas 3.4 and 2.1 we derive the following comparison lemma:

Lemma 3.5. Let $u \in C^{2}(\Omega)$ be a strictly convex solution of (1.1). Suppose that $\psi$ satisfies (1.3) with $p>n$ and that $\Omega$ contains a ball $B_{a}\left(x_{0}\right)$. Then $u(x) \leq w^{a, M}\left(x-x_{0}\right)$ for all $x \in \Omega$.

The second inequality in (1.6) now follows from Lemma 3.5:

Corollary 3.6. Let $u \in C^{2}(\Omega)$ be a strictly convex solution of (1.1) where $\Omega$ is a convex (not necessarily bounded) domain in $\mathbb{R}^{n}$. Suppose $\psi$ satisfies (1.3) with $p>n$ and $M>0$. Then

$$
u(x) \leq \bar{h}(d(x)), \quad \forall x \in \Omega,
$$

where $d$ is the distance function to $\partial \Omega$ and $\bar{h} \in C^{\infty}\left(\mathbb{R}^{+}\right)$is given by

$$
\bar{h}(r):=w^{r, M}(0), \quad r>0 .
$$

We next construct subsolutions to (1.1) defined on the whole space $\mathbb{R}^{n}$ when $\psi$ satisfies (1.9) with $p+q \leq n$.

Lemma 3.7. Assume $p, q \geq 0, p+q \leq n$ and $M>0$. Then there exists a strictly convex radially symmetric positive function $\widetilde{u} \in C^{\infty}\left(\mathbb{R}^{n}\right)$ satisfying

$$
\operatorname{det} D^{2} \widetilde{u}(x) \geq M\left(1+(\widetilde{u}(x))^{p}\right)\left(1+|D \widetilde{u}(x)|^{q}\right), \quad \forall x \in \mathbb{R}^{n} .
$$

Proof. Without loss of generality we may assume $M=1$. Let us consider separately three cases: $q=0, q=n$ and $0<q<n$.

Case i: $q=0$. Consider the initial value problem

$$
\begin{aligned}
\varphi^{\prime} & =r\left(1+\varphi^{p}\right)^{1 / n}, \quad r>0, \\
\varphi(0) & =1 .
\end{aligned}
$$


It is easy to see that when $p \leq n$ there exists a unique smooth solution $\varphi$ to (3.8) defined for all $r \geq 0$ and strictly increasing. Indeed, suppose $\varphi$ is defined on $[0, R)$. For any $\rho<R$, by (3.8) we have

$$
\begin{aligned}
\rho^{2} & =2 \int_{0}^{\rho} r d r=2 \int_{0}^{\rho} \frac{\varphi^{\prime}(r)}{\left(1+(\varphi(r))^{p}\right)^{1 / n}} d r \\
& \geq \int_{0}^{\rho} \frac{\varphi^{\prime}(r)}{(\varphi(r))^{p / n}} d r= \begin{cases}\log \varphi(\rho), & p=n ; \\
\frac{n}{n-p}(\varphi(\rho))^{(n-p) / n}, & p<n .\end{cases}
\end{aligned}
$$

It follows that $\lim _{\rho \rightarrow R} \varphi(\rho)=+\infty$ if and only if $R=+\infty$. We rewrite (3.8) in the form

$$
\left(\varphi^{\prime}\right)^{n}=r^{n}\left(1+\varphi^{p}\right)
$$

and take derivatives of both sides to obtain

$$
\left(\varphi^{\prime}\right)^{n-1} \varphi^{\prime \prime} \geq r^{n-1}\left(1+\varphi^{p}\right) .
$$

By (3.1) we see that the function $\widetilde{u}(x):=\varphi(|x|)$ is strictly convex and

$$
\operatorname{det} D^{2} \widetilde{u} \geq\left(1+\widetilde{u}^{p}\right) \text { in } \mathbb{R}^{n} .
$$

Case ii: $q=n$. In this case $p=0$. Let $\varphi \in C^{\infty}\left(\mathbb{R}^{+}\right)$be given by

$$
\varphi(r):=\int_{0}^{r}\left(\exp \left(r^{n}\right)-1\right)^{1 / n} d r, \quad r \geq 0 .
$$

Then

$$
\varphi^{\prime}(r)=\left(\exp \left(r^{n}\right)-1\right)^{1 / n}>0, \quad r>0 .
$$

Moreover $\varphi$ is strictly convex and $\varphi(0)=\varphi^{\prime}(0)=0$. Rewriting (3.9) as

$$
\log \left(1+\left(\varphi^{\prime}\right)^{n}\right)=r^{n}
$$

and taking derivatives, we obtain

$$
\left(\varphi^{\prime}\right)^{n-1} \varphi^{\prime \prime}=r^{n-1}\left(1+\left(\varphi^{\prime}\right)^{n}\right) .
$$

Consequently, the function $\widetilde{u}(x):=1+\varphi(|x|), x \in \mathbb{R}^{n}$, which is smooth and strictly convex, satisfies

$$
\operatorname{det} D^{2} \widetilde{u}=\left(1+|D \widetilde{u}|^{n}\right) \text { in } \mathbb{R}^{n} .
$$

Case iii: $0<q<n$. Let $\varphi$ be the solution defined in some interval $[0, R)$ of the initial value problem

$$
\begin{aligned}
\varphi^{\prime} & =\left(\left(1+r^{n}\left(1+\varphi^{p}\right)\right)^{n / n-q}-1\right)^{1 / n}, \quad r>0 \\
\varphi(0) & =1 .
\end{aligned}
$$


Then $\varphi^{\prime}(0)>0$ and $\varphi^{\prime}(r)>0$ for $r>0$. Moreover,

$$
\begin{aligned}
\varphi^{\prime} & \leq\left(1+r^{n}\left(1+\varphi^{p}\right)\right)^{1 /(n-q)} \\
& \leq\left(1+\varphi^{p}\right)^{1 /(n-q)}\left(1+r^{n}\right)^{1 /(n-q)} \leq c \varphi^{p /(n-q)}\left(1+r^{n}\right)^{1 /(n-q)} .
\end{aligned}
$$

Since $p+q \leq n$, we see that $\varphi$ is defined for all $r \geq 0$. Rewriting (3.10) as

$$
\left(1+\left(\varphi^{\prime}\right)^{n}\right)^{(n-q) / n}=1+r^{n}\left(1+\varphi^{p}\right)
$$

we obtain by differentiation

$$
\begin{aligned}
(n-q)\left(\varphi^{\prime}\right)^{n-1} \varphi^{\prime \prime} & =r^{n-1}\left(1+\varphi^{p}\right)\left(1+\left(\varphi^{\prime}\right)^{n}\right)^{q / n} \\
& \geq \frac{1}{2} r^{n-1}\left(1+\varphi^{p}\right)\left(1+\left(\varphi^{\prime}\right)^{q}\right) .
\end{aligned}
$$

Consequently the function $\widetilde{u}(x):=c \varphi(|x|)$, where $c$ is a constant, is smooth, strictly convex and satisfies (3.7) when $c$ is large enough.

We conclude this section with proofs of Theorems 1.4-1.6.

Proof of Theorem 1.4. Let $u \in C^{2}(\Omega)$ be a convex solution of (1.1)-(1.2), where $\Omega$ is bounded and $\psi$ satisfies (1.9). Let $\widetilde{u} \in C^{\infty}\left(\mathbb{R}^{n}\right)$ satisfy (3.7) in Lemma 3.7. Note that $u-C \widetilde{u}=\infty$ on $\partial \Omega$ for any $C>0$. Since $\widetilde{u}>0$, we can choose $C>1$ such that

$$
u(y)-C \widetilde{u}(y)=\min _{\Omega}(u-C \widetilde{u})<0
$$

for some $y \in \Omega$.

By (1.9) we have

$$
\begin{aligned}
\operatorname{det} D^{2} u(y) & \leq M\left(1+\left(u^{+}(y)\right)^{p}\right)\left(1+|D u(y)|^{q}\right) \\
& \leq M\left(1+(C \widetilde{u}(y))^{p}\right)\left(1+C^{q}|D \widetilde{u}(y)|^{q}\right) \\
& <C^{n} M\left(1+(\widetilde{u}(y))^{p}\right)\left(1+|D \widetilde{u}(y)|^{q}\right) \leq C^{n} \operatorname{det} D^{2} \widetilde{u}(y),
\end{aligned}
$$

since $C>1, p+q \leq n$ and $D u(y)=D \widetilde{u}(y)$, contradicting the fact that $D^{2}(u-C \widetilde{u})(y)$ is a positive semidefinite matrix. The proof is complete.

Proof of Theorem 1.6. We follow an idea of Cheng and Yau [6]. Assume $\Omega$ contains the line

$$
L: x_{1}=\cdots=x_{n-1}=0 .
$$

Since $\Omega$ is convex, it contains a solid cylinder $\left\{x:=\left(x^{\prime}, x_{n}\right) \in \mathbb{R}^{n}:\left|x^{\prime}\right|<\delta\right\}$, for some $\delta>0$, where $x^{\prime}=\left(x_{1}, \ldots, x_{n-1}\right)$. For any $\lambda>0$, let $E_{\lambda}$ be the ellipsoid

$$
\frac{\left|x^{\prime}\right|^{2}}{\delta^{2}}+\frac{x_{n}^{2}}{(\delta \lambda)^{2}} \leq 1
$$

and consider the function

$$
w_{\lambda}(x):=\lambda^{\alpha} w^{\delta, M}\left(x^{\prime}, \lambda^{-1} x_{n}\right), \quad x \in E_{\lambda}
$$


where $\alpha=2 /(n-p)$ and $w^{\delta, M}$ is as in Lemma 3.4. We have

$$
\begin{aligned}
\operatorname{det} D^{2} w_{\lambda}(x) & =\lambda^{n \alpha-2} \operatorname{det} D^{2} w^{\delta, M}\left(x^{\prime}, \lambda^{-1} x_{n}\right) \\
& \leq M\left(\lambda^{\alpha} w^{\delta, M}\left(x^{\prime}, \lambda^{-1} x_{n}\right)\right)^{p}=M\left(w_{\lambda}(x)\right)^{p}, \quad \forall x \in E_{\lambda} .
\end{aligned}
$$

Now assume that $u \in C^{2}(\Omega)$ is a convex solution of (1.1)-(1.2) in $\Omega$, where $\psi$ satisfies (1.3). Since $w_{\lambda}=+\infty$ on $\partial E_{\lambda} \subset \Omega$, Lemma 2.1 yields

$$
w_{\lambda} \geq u \text { in } E_{\lambda} .
$$

Note that $\alpha<0$. Letting $\lambda \rightarrow \infty$, we see that $u=0$ on $L$. It follows that $u_{x_{n} x_{n}}=0$ on $L$, contradicting the positivity of $\operatorname{det} D^{2} u$ everywhere in $\Omega$.

Finally, Theorem 1.5 follows from the comparison principle (Lemma 2.1) and the following lemma:

Lemma 3.8. Let $\alpha>1$ and $a>0$. There exists a strictly convex radially symmetric function $\bar{u} \in C^{2}\left(B_{a}(0)\right)$ satisfying

$$
\begin{aligned}
\operatorname{det} D^{2} \bar{u} & =\frac{1}{a^{n}(\alpha-1)}\left(1+|D \bar{u}|^{n}\right)^{\alpha} \text { in } B_{a}(0), \\
\frac{\partial \bar{u}}{\partial \nu} & =+\infty \text { on } \partial B_{a}(0),
\end{aligned}
$$

where $\nu$ is the unit normal to $\partial B_{a}(0)$. Moreover, if $\alpha>(n+1) / n$ then $\bar{u} \in C^{0}\left(\overline{B_{a}(0)}\right)$.

Proof. Let $\beta>0$ and consider the function $\varphi$ defined by

$$
\varphi(r):=\int_{0}^{r}\left(\left(1-r^{n}\right)^{-\beta}-1\right)^{1 / n} d r, \quad 0 \leq r<1 .
$$

Then

$$
1+\left(\varphi^{\prime}\right)^{n}=\frac{1}{\left(1-r^{n}\right)^{\beta}}
$$

and

$$
\left(\varphi^{\prime}\right)^{n-1} \varphi^{\prime \prime}=\beta r^{n-1}\left(1+\left(\varphi^{\prime}\right)^{n}\right)^{(\beta+1) / \beta} .
$$

We see that $\varphi(0)=\varphi^{\prime}(0)=0$, that $\varphi^{\prime \prime}(r)>0$ for all $0 \leq r<1$ and that $\lim _{r \rightarrow 1} \varphi^{\prime}(r)=\infty$. Note also that if $\beta<n$,

$$
\varphi(r) \leq \int_{0}^{r}\left(1-r^{n}\right)^{-\beta / n} d r \leq \int_{0}^{r}(1-r)^{-\beta / n} d r \leq \frac{n}{n-\beta}, \quad \forall r<1 .
$$

Taking $\beta=1 /(\alpha-1)$, we obtain the desired function $\bar{u}(x):=a \varphi\left(a^{-1}|x|\right)$, for $x \in B_{a}(0)$. 


\section{Proof of Theorem 1.1}

By assumption (1.5) we may find a positive nondecreasing function $\eta$ in $C^{\infty}\left(\mathbb{R}^{n}\right)$ satisfying

$$
e^{\varepsilon z} \eta(z) \geq \max _{y \leq z} \Psi(y), \quad \forall z \in \mathbb{R} .
$$

For simplicity we will assume throughout this section that $\varepsilon=1$; this may be achieved by rescaling.

We first assume $\Omega$ to be smooth. For each integer $k \geq 1$, consider the Dirichlet problem

$$
\begin{aligned}
\operatorname{det} D^{2} u & =\psi(x, u, D u)>0 \text { in } \Omega, \\
u & =k \text { on } \partial \Omega .
\end{aligned}
$$

Since $\Omega$ is bounded, we may choose $r>0$ sufficiently large that $\Omega \subset B_{r}(0)$ and $v^{r, \eta} \leq 1$ on $\partial \Omega$. It follows from Lemma 2.1 that $v^{r, \eta} \leq u \leq k$ in $\bar{\Omega}$, so

$$
|u| \leq C_{k}
$$

for any convex solution $u$ of (4.2), where $C_{k}$ is a constant depending on $k$. By a result of Lions [10] (see also [4]), there exists for each $k$ a strictly convex function $\underline{u}_{k} \in C^{2}(\bar{\Omega})$ satisfying

$$
\begin{aligned}
\operatorname{det} D^{2} \underline{u}_{k} & \geq \Psi\left(C_{k}\right)\left(1+\left|D u_{k}\right|^{n}\right) \text { in } \Omega, \\
\underline{u}_{k} & =k \text { on } \partial \Omega .
\end{aligned}
$$

Note that $\underline{u}_{k}$ is a subsolution of of (4.2). By a theorem of CaffarelliNirenberg-Spruck [4] there exists a strictly convex solution $u_{k} \in C^{\infty}(\bar{\Omega})$ of (4.2) satisfying $u_{k} \geq \underline{u}_{k}$ in $\bar{\Omega}$ for each $k \geq 1$. Moreover, $u_{k}$ satisfies the a priori estimate

$$
\left\|u_{k}\right\|_{C^{2, \alpha}(\bar{\Omega})} \leq C(k), \quad k \geq 1,
$$

where $C(k)>0$ depends on $k$. We next need to derive a priori interior estimates which are independent of $k$.

Proposition 4.1. For an arbitrary compact subset $K$ of $\Omega$, there exists a constant $C$ independent of $k$ such that

$$
\left\|u_{k}\right\|_{C^{2, \alpha}(K)} \leq C, \quad \forall k \geq 1 .
$$

The proof of this estimate is based on the following lemma and some well-known results in the theory of Monge-Ampère and more general fully nonlinear elliptic equations.

Lemma 4.2. There exists $a>0$ depending only on $\Omega$ and a decreasing sequence $a_{k} \rightarrow a(k \rightarrow \infty)$ such that

$$
v^{a_{k}, \eta}(a-d(x)) \leq u_{k}(x) \leq \bar{h}(d(x)), \quad \forall x \in \Omega, k \geq 1,
$$


where $d$ is the distance function to the boundary of $\Omega$. (For the definitions of $\bar{h}$ and $v^{a_{k}, \eta}$, see (3.6) and Remark 3.2.)

Proof. The second inequality follows from Corollary 3.6. Next, let $a>0$ be the smallest number such that for any point $\bar{x} \in \partial \Omega$ there is a ball $B_{a}\left(x_{0}\right)$ of radius $a$ with $\Omega \subset B_{a}\left(x_{0}\right)$ and $\bar{\Omega} \cap \partial B_{a}\left(x_{0}\right)=\{\bar{x}\}$; such a number exists since $\Omega$ is bounded and strictly convex. Choose $a_{1}>a_{2}>\cdots>a_{k}>\cdots$, $a_{k} \rightarrow a$ as $k \rightarrow \infty$, such that $v^{a_{k}, \eta}(a)=k$ for each $k \geq 1$. For $x \in \Omega$, choose $\bar{x} \in \partial \Omega$ and a ball $B_{a}\left(x_{0}\right)$ such that $d(x)=|x-\bar{x}|, \Omega \subset B_{a}\left(x_{0}\right)$ and $\bar{\Omega} \cap \partial B_{a}\left(x_{0}\right)=\{\bar{x}\}$. Since $v^{a_{k}, \eta}\left(x-x_{0}\right) \leq u_{k}(x)$ for all $x \in \partial \Omega$, Lemma 2.1 gives

$$
v^{a_{k}, \eta}\left(x-x_{0}\right) \leq u_{k}(x), \quad \forall x \in \Omega .
$$

This proves the first inequality in (4.6).

For convenience let us now introduce some notation. Let $h, v_{k}$ denote the functions defined in $\Omega$ by

$$
h(x):=\bar{h}(d(x)), \quad v_{k}(x):=v^{a_{k}, \eta}(a-d(x)), \quad x \in \Omega .
$$

For $l>0$ and $k \geq 1$ write

$$
\begin{aligned}
H_{l} & :=\{x \in \Omega: h(x)<l\}, \\
U_{k, l} & :=\left\{x \in \Omega: u_{k}(x)<l\right\}, \\
V_{k, l} & :=\left\{x \in \Omega: v_{k}(x)<l\right\} .
\end{aligned}
$$

By (4.6) we have $H_{l} \subset U_{k, l} \subset V_{k, l}$ for each $k \geq 1$.

Proof of Proposition 4.1. Let $K$ be a compact subset of $\Omega$. We may choose $l>0$ and then $k_{0}$ sufficiently large so that $K \subset H_{l / 2}$ and $\bar{V}_{k_{0}, 4 l} \subset \Omega$. From (4.6) we see that

$$
\left|u_{k}\right| \leq C_{0} \text { in } \bar{U}_{k, 2 l}, \quad \forall k \geq k_{0},
$$

where $C_{0}$ is independent of $k$. Moreover, by the strict convexity of $u_{k}$,

$$
\begin{aligned}
\max _{\bar{U}_{k, 2 l}}\left|D u_{k}\right|=\max _{\partial U_{k, 2 l}}\left|D u_{k}\right| & \leq \max _{x \in \partial V_{k_{0}, 4 l}} \frac{u_{k}(x)-2 l}{\operatorname{dist}\left(\bar{U}_{k, 2 l}, \partial V_{k_{0}, 4 l}\right)} \\
& \leq \max _{x \in \partial V_{k_{0}, 4 l}} \frac{h(x)-2 l}{\operatorname{dist}\left(\bar{V}_{k, 2 l}, \partial V_{k_{0}, 4 l}\right)} \\
& \leq \max _{x \in \partial V_{k_{0}, 4 l}} \frac{h(x)-2 l}{\operatorname{dist}\left(\bar{V}_{k_{0}, 2 l}, \partial V_{k_{0}, 4 l}\right)} \equiv C_{1}
\end{aligned}
$$

for all $k \geq k_{0}$, where the last two inequalities follow from the relations $u_{k}<h$ and $U_{k, 2 l} \subset V_{k, 2 l} \subset V_{k_{0}, 2 l}$, since $v_{k} \geq v_{k_{0}}$ for $k \geq k_{0}$.

Next, applying Pogorelov's interior estimates (see [7]) we obtain

$$
\left|D^{2} u_{k}(x)\right| \leq \frac{C_{2}}{\operatorname{dist}\left(x, \partial U_{k, 2 l}\right)}, \quad \forall x \in U_{k, 2 l}, k \geq k_{0},
$$


where $C_{2}$ depends on $C_{0}, C_{1}$ and the $C^{2}$ norm of $\psi$, as well as $\min \psi$, in $\bar{\Omega} \times\left\{z \leq C_{0}\right\} \times\left\{|\mathbf{p}| \leq C_{1}\right\}$, but is independent of $k$. Since $H_{l} \subset H_{2 l} \subset U_{k, 2 l}$,

$$
\operatorname{dist}\left(H_{l}, \partial U_{k, 2 l}\right) \geq \operatorname{dist}\left(H_{l}, \partial H_{2 l}\right) .
$$

It follows from (4.9) that

$$
\left\|D^{2} u_{k}\right\|_{C^{0}\left(\bar{H}_{l}\right)} \leq \frac{C_{2}}{\operatorname{dist}\left(H_{l}, \partial H_{2 l}\right)} .
$$

Finally, by the Evans-Krylov theorem (see [3]) we have

$$
\left\|D^{2} u_{k}\right\|_{C^{\alpha}\left(\bar{H}_{l / 2}\right)} \leq C_{3}, \quad \forall k \geq k_{0}
$$

where $C_{3}$ is independent of $k$. Now (4.5) follows from (4.7), (4.8), (4.10) and (4.11), combining with (4.4) for $k \leq k_{0}$.

By Proposition 4.1, there exists a subsequence $\left\{u_{k_{j}}\right\}$ and $u \in C^{2, \alpha}(\Omega)$ such that

$$
\lim _{j \rightarrow \infty}\left\|u_{k_{j}}-u\right\|_{C^{2, \alpha}(K)}=0
$$

for any compact subset $K$ of $\Omega$. We see that $u$ is strictly convex and solves (1.1). From (4.6) we obtain

$$
\underline{h}\left(d(x):=v^{a, \eta}(a-d(x)) \leq u(x) \leq \bar{h}(d(x)), \quad \forall x \in \Omega .\right.
$$

Consequently, $u=+\infty$ on $\partial \Omega$. This completes the proof of Theorem 1.1 when $\Omega$ is smooth.

Suppose now that $\Omega$ is not smooth. We choose a sequence of smooth strictly convex domains

$$
\Omega_{1} \subseteq \cdots \subseteq \Omega_{k} \subseteq \Omega_{k+1} \subseteq \cdots \subseteq \Omega
$$

such that

$$
\Omega=\bigcup_{k=1}^{\infty} \Omega_{k} .
$$

For each $k \geq 1$, let $u_{k} \in C^{\infty}\left(\Omega_{k}\right)$ be a strictly convex solution of the problem

$$
\begin{aligned}
\operatorname{det} D^{2} u & =\psi(x, u, D u) \text { in } \Omega_{k}, \\
u & =\infty \text { on } \partial \Omega_{k} .
\end{aligned}
$$

We have

$$
v^{a, \eta}(a-d(x)) \leq u_{k}(x) \leq \bar{h}\left(d_{k}(x)\right), \quad \forall x \in \Omega_{k},
$$

where $a$ is as in (4.12) and $d_{k}$ is the distance function to $\partial \Omega_{k}$. Using this in place of Lemma 4.2 we can derive the estimate (4.5) as before, and therefore obtain a subsequence that converges to a solution $u \in C^{2, \alpha}(\Omega)$ of (1.1)(1.2) satisfying (4.12). That $u$ lies in $C^{\infty}(\Omega)$ follows from elliptic regularity theory. The proof of Theorem 1.1 is complete. 
Remark 4.3. As an alternative approach, one may first prove the existence of a convex weak solution and then apply the strict convexity and regularity theorems of Caffarelli [1], [2] to prove Theorem 1.1.

\section{Proof of Theorem 1.2}

The proof of Theorem 1.2 follows that of Theorem 1.1, except that we have to reconstruct lower barriers when $\Omega$ is unbounded or not strictly convex. To this end we consider the equation

$$
\operatorname{det} D^{2} u=F(u) \text { in } \Gamma^{+}:=\left\{x \in \mathbb{R}^{n}: x_{i}>0\right\},
$$

where $F$ is a positive nondecreasing function. When $F(u)=e^{2 u}$, Cheng and Yau [6] observed that $u(x):=-\log \left(x_{1} \ldots x_{n}\right)$ is a strictly convex solution of (5.1) in $\Gamma^{+}$. Inspired by this we look for solutions to (5.1) of the form

$$
u(x)=\varphi\left(a-\log \left(x_{1} \ldots x_{n}\right)\right), \quad x=\left(x_{1}, \ldots, x_{n}\right) \in \Gamma^{+},
$$

for some function $\varphi$, where $a$ is a constant. We calculate

$$
u_{x_{i}}=\frac{-\varphi^{\prime}}{x_{i}}, \quad u_{x_{i} x_{j}}=\frac{1}{x_{i} x_{j}}\left(\varphi^{\prime \prime}+\varphi^{\prime} \delta_{i j}\right) .
$$

It follows that

$$
\operatorname{det} D^{2} u=\frac{1}{\left(x_{1} \ldots x_{n}\right)^{2}}\left(\varphi^{\prime}\right)^{n-1}\left(n \varphi^{\prime \prime}+\varphi^{\prime}\right) .
$$

Equation (5.1) thus reduces to

$$
\left(\varphi^{\prime}\right)^{n-1}\left(n \varphi^{\prime \prime}+\varphi^{\prime}\right)=e^{2(a-t)} F(\varphi) .
$$

Lemma 5.1. Let $a>0$ and $F \in C^{\infty}(\mathbb{R})$ satisfy $F>0, F^{\prime} \geq 0$ and

$$
F(z) \geq M\left(z^{+}\right)^{p}, \quad \forall z \in \mathbb{R},
$$

where $p>n$. There exists a strictly increasing function $\underline{\varphi} \in C^{\infty}\left(\mathbb{R}^{+}\right)$with

$$
\left(\underline{\varphi}^{\prime}\right)^{n-1}\left(n \underline{\varphi}^{\prime \prime}+\underline{\varphi}^{\prime}\right) \geq e^{2(a-t)} F(\underline{\varphi}(t)), \quad \forall t \geq 0,
$$

and

$$
\lim _{t \rightarrow+\infty} \underline{\varphi}(t)=+\infty
$$

Proof. We construct $\varphi$ from $F$. For convenience we write $f:=A\left(e^{2 a} F\right)^{1 / n}$, where $A$ is an undetermined constant, and define

$$
g(z):=\int_{0}^{z} \frac{d z}{f(z)} .
$$

We see that $g$ is a strictly increasing function defined for all $z \in \mathbb{R}$. Let $g^{-1}$ denote the inverse function of $g$ and define

$$
\underline{\varphi}(t):=g^{-1}\left(B-e^{-\beta t}\right),
$$


where $\beta$ is a constant to be determined and

$$
B:=\int_{0}^{\infty} \frac{d z}{f(z)}<\infty
$$

by assumption (5.3). It is clear that $\varphi$ satisfies (5.5). We calculate

$$
\underline{\varphi}^{\prime}(t)=\frac{\beta e^{-\beta t}}{g^{\prime}(\underline{\varphi}(t))}=\beta e^{-\beta t} f(\underline{\varphi}(t))>0, \quad \forall t \in \mathbb{R},
$$

and

$$
\begin{aligned}
\underline{\varphi}^{\prime \prime}(t) & =\beta e^{-\beta t}\left(f^{\prime}(\underline{\varphi}(t)) \underline{\varphi}^{\prime}(t)-\beta f(\underline{\varphi}(t))\right) \\
& =\beta^{2} e^{-\beta t} f(\underline{\varphi}(t))\left(e^{-\beta t} f^{\prime}(\underline{\varphi}(t))-1\right) \geq-\beta^{2} e^{-\beta t} f(\underline{\varphi}(t)),
\end{aligned}
$$

since $f^{\prime}(\underline{\varphi}(t)) \geq 0$. It follows that

$$
\left(\underline{\varphi}^{\prime}\right)^{n-1}\left(n \underline{\varphi}^{\prime \prime}+\underline{\varphi}^{\prime}\right) \geq \beta^{n}(1-n \beta) e^{-n \beta t}(f(\underline{\varphi}(t)))^{n} .
$$

Taking $\beta<1 / n$ and $A=\beta^{-1}(1-n \beta)^{-1 / n}$ we obtain (5.4).

A slight modification of this proof yields the following:

Lemma 5.2. Let $a>0$ and $F(z)=e^{\varepsilon z} \eta(z)$, where $\varepsilon>0$ and $\eta \in C^{\infty}(\mathbb{R})$ is a positive nondecreasing function. There exists a strictly increasing function $\underline{\varphi} \in C^{\infty}\left(\mathbb{R}^{+}\right)$satisfying (5.4) for all $t \in \mathbb{R}$ and (5.5). Moreover, $\underline{\varphi}$ is a convex function.

Proof. As in the proof of Lemma 5.1 we define $\varphi$ by (5.7). Note that here we still have $B:=\int_{0}^{\infty} d z / f(z)<+\infty$. Write $s=\underline{\varphi}(t)$. By (5.7) we have

$$
e^{-\beta t}=B-g(s)=\int_{s}^{\infty} \frac{d z}{f(z)} \geq \frac{1}{A\left(e^{2 a} \eta(s)\right)^{1 / n}} \int_{s}^{\infty} e^{-\varepsilon z / n} d z=\frac{n}{\varepsilon f(s)},
$$

since $\eta$ is nondecreasing. Next,

$$
f^{\prime}(s)=\frac{A^{n} e^{2 a} F^{\prime}(s)}{n(f(s))^{n-1}}=\frac{A^{n} e^{2 a} e^{\varepsilon s}\left(\varepsilon \eta(s)+\eta^{\prime}(s)\right)}{n(f(s))^{n-1}} \geq \frac{\varepsilon A^{n} e^{2 a} F(s)}{n(f(s))^{n-1}}=\frac{\varepsilon f(s)}{n},
$$

since $\eta^{\prime} \geq 0$. Consequently, $\underline{\varphi}^{\prime \prime}(t) \geq 0$ by (5.8). Finally, taking $\beta=A^{-1}=\frac{2}{n}$ we have

$$
\left(\underline{\varphi}^{\prime}\right)^{n-1}\left(n \underline{\varphi}^{\prime \prime}+\underline{\varphi}^{\prime}\right) \geq\left(\underline{\varphi}^{\prime}(t)\right)^{n}=\beta^{n} e^{-n \beta t}(f(\underline{\varphi}(t)))^{n}=e^{2(a-t)} F(\underline{\varphi}(t))
$$

for all $t \in \mathbb{R}$.

Remark 5.3. Let $\varphi$ be the unique solution of (5.2) satisfying the initial data

$$
\varphi(0)=\underline{\varphi}(0), \quad \varphi^{\prime}(0)=\underline{\varphi}^{\prime}(0) .
$$


We have $\varphi(t) \leq \underline{\varphi}(t)$ for all $t>0$ where $\varphi(t)$ is defined. Equation (5.2) can be recast as

$$
\left(e^{t}\left(\varphi^{\prime}\right)^{n}\right)^{\prime}=e^{2 a-t} F(\varphi),
$$

so $0<\varphi^{\prime}(t) \leq \varphi^{\prime}(t)$ for all $t>0$ where $\varphi(t)$ is defined. By the extension theorem we see that $\varphi$ is defined for all $t>0$. However, $\varphi$ may be bounded above on all of $\mathbb{R}^{+}$, so we cannot replace $\varphi$ by $\varphi$ in the construction below.

Proof of Theorem 1.2. As in the last part of the proof of Theorem 1.1 we choose a sequence of bounded smooth strictly convex domains $\Omega_{1} \subseteq \Omega_{2} \subseteq$ $\cdots \subseteq \Omega_{k} \subseteq \cdots \subseteq \Omega$ such that $\Omega=\bigcup \Omega_{k}$ and we consider, for each $k$,

$$
\begin{aligned}
\operatorname{det} D^{2} u & =\psi(x, u) \text { in } \Omega_{k}, \\
u & =k \text { on } \partial \Omega_{k} .
\end{aligned}
$$

Let $u_{k} \in C^{\infty}\left(\Omega_{k}\right)$ be a strictly convex solution of (5.12); the existence of $u_{k}$ follows from [4]. By assumption (1.3) and Corollary 3.6 we have

$$
u_{k}(x) \leq \bar{h}\left(d_{k}(x)\right), \quad \forall x \in \Omega_{k},
$$

where $d_{k}$ is the distance function to $\partial \Omega_{k}$. We need an a priori lower bound for $u_{k}$, which is derived below (Lemma 5.4). With the aid of such estimates, the rest of proof proceeds as that of Theorem 1.1.

Lemma 5.4. There exists an increasing sequence of functions $\underline{h}_{k} \in C\left(\mathbb{R}^{+}\right)$ such that

$$
\lim _{k \rightarrow \infty} \lim _{r \rightarrow 0} \underline{h}_{k}(r)=+\infty
$$

and

$$
u_{k}(x) \geq \underline{h}_{k}(d(x)), \quad \forall x \in \Omega_{k},
$$

for all $k$ sufficiently large, where $d(x)=\operatorname{dist}(x, \partial \Omega)$.

Proof. By assumption (1.7) we may find a function $\eta \in C^{\infty}(\mathbb{R})$ with $\eta>0$, $\eta^{\prime} \geq 0$ and $F(z):=e^{\varepsilon z} \eta(z) \geq \psi(x, z)$ for all $(x, z) \in \bar{\Omega} \times \mathbb{R}$, where $\varepsilon \geq 0$ as in Theorem 1.2. We consider two cases.

Case i: $\varepsilon>0$. We apply Lemma 5.2 with $a=0$ to obtain $\underline{\varphi} \in C^{\infty}(\mathbb{R})$ satisfying (5.4) and (5.5). By the assumption that $\Omega$ contains no straight lines we may assume $\Omega \subset \Gamma^{+}=\left\{x \in \mathbb{R}^{n}: x_{i}>0\right\}$. For a fixed point $x_{0} \in \Omega$ let $\bar{x}$ be a point on $\partial \Omega$ such that $d\left(x_{0}\right)=\operatorname{dist}\left(x_{0}, \bar{x}\right)$. We may assume $\bar{x}$ lies on the hyperplane $x_{1}=0$. For each integer $k \geq 1$ let

$$
\underline{u}_{k}(x):=\underline{\varphi}\left(-\log \left(\left(x_{1}+b_{k}\right) \cdots\left(x_{n}+b_{k}\right)\right)\right), x \in \Gamma^{+},
$$

where $b_{k}$ satisfies $\underline{\varphi}\left(-n \log b_{k}\right)=k$. Then $\underline{u}_{k} \in C^{\infty}\left(\Gamma^{+}\right)$is strictly convex and

$$
\operatorname{det} D^{2} \underline{u}_{k}(x) \geq F\left(\underline{u}_{k}(x)\right) \geq \psi\left(x, \underline{u}_{k}(x)\right), \quad x \in \Omega \text {. }
$$


Note that $\underline{u}_{k} \leq u_{k}$ on $\partial \Omega_{k}$. By Lemma 2.1 we obtain

$$
\underline{u}_{k} \leq u_{k} \text { in } \Omega \text {. }
$$

In particular, $\underline{u}_{k}\left(x_{0}\right) \leq u_{k}\left(x_{0}\right)$ if $k$ is sufficiently large and $x_{0} \in \Omega_{k}$. The function

$$
\underline{h}_{k}(r):=\min _{|x-\bar{x}|=r, x \in \Gamma^{+}} \underline{u}_{k}(x)
$$

then has the desired properties.

Case ii: $\varepsilon=0$ and $\Omega$ is bounded. We may assume that

$$
\Omega \subseteq Q:=\left\{x \in \mathbb{R}^{n}: 0<x_{i}<\rho, 1 \leq i \leq n\right\} \subset \mathbb{R}^{n}
$$

and $\bar{x}=\left(0, \frac{1}{2} \rho, \ldots, \frac{1}{2} \rho\right)$, where $\rho$ is the diameter of $\Omega$. Applying Lemma 5.1 to $F$ with $a=a_{k}:=n \log \left(\rho+b_{k}\right)$, where $b_{k}>0$ is to be determined, we obtain $\underline{\varphi}_{k} \in C^{\infty}\left(\mathbb{R}^{+}\right)$satisfying (5.4) for $t \geq 0$ and (5.5). Let

$$
\underline{u}_{k}(x):=\underline{\varphi}_{k}\left(a_{k}-\log \left(\left(x_{1}+b_{k}\right) \cdots\left(x_{n}+b_{k}\right)\right)\right), \quad x \in Q,
$$

and choose a decreasing sequence $b_{k}$ such that $\underline{\varphi}_{k}\left(a_{k}-n \log b_{k}\right) \leq k$ for all $k$ sufficiently large. We now can proceed as in the previous case.

This completes the proof of Theorem 1.2. Finally, it is clear that with minor modifications the proof yields Theorems 1.1 and 1.2 with assumption (1.8) in place of (1.3) when $\psi_{z} \geq 0$. (See Remark 1.3.)

Acknowledgements. Part of this work was done while the second author was visiting the University of Tennessee. He wishes to thank the Department of Mathematics and the University for their hospitality.

\section{References}

[1] L.A. Caffarelli, A localization property of viscosity solutions to the Monge-Ampère equation and their strict convexity, Ann. of Math., 131 (1990), 129-134, MR 1038359 (91f:35058), Zbl 0704.35045.

[2] L. A. Caffarelli, Interior $W^{2, p}$ estimates for solutions of the Monge-Ampère equation, Ann. of Math., 131 (1990), 135-150, MR 1038360 (91f:35059), Zbl 0704.35044.

[3] L.A. Caffarelli and X. Cabré, Fully Nonlinear Elliptic Equations, AMS Colloquium Publications 43, American Mathematical Society, Providence, 1995, MR 1351007 (96h:35046), Zbl 0834.35002.

[4] L.A. Caffarelli, L. Nirenberg and J. Spruck, The Dirichlet problem for nonlinear second-order elliptic equations I. Monge-Ampère equations, Comm. Pure Appl. Math., 37 (1984), 369-402, MR 0739925 (87f:35096), Zbl 0598.35047.

[5] S.Y. Cheng and S.-T. Yau, On the existence of a complete Kähler metric on noncompact complex manifolds and the regularity of Fefferman's equation, Comm. Pure Appl. Math., 33 (1980), 507-544, MR 0575736 (82f:53074), Zbl 0506.53031. 
[6] S. Y. Cheng and S. T. Yau, The real Monge-Ampère equation and affine flat structure, Proc. 1980 Beijing Symp. on Diff. Geom. and Diff. Equations, Vol. I (S.S. Chern and W.T. Wu, eds.), Science Press, Beijing, 1982, 339-370, MR 0714338 (85c:53103), Zbl 0517.35020.

[7] D. Gilbarg and N.S. Trudinger, Elliptic Partial Differential Equations of Second Order, Grundlehren der Math. Wissenschaften 224, Springer-Verlag, Berlin, 1983, MR 0737190 (86c:35035), Zbl 0562.35001.

[8] J.B. Keller, On solutions of $\Delta u=f(u)$, Comm. Pure Appl. Math., 10 (1957), 503510, MR 0091407 (19,964c), Zbl 0090.31801.

[9] A.C. Lazer and P.J. McKenna, On singular boundary value problems for the Monge-Ampère operator, J. Math. Anal. Appl., 197 (1996), 341-362, MR 1372183 (97c:35064), Zbl 0856.35042.

[10] P.-L. Lions, Sur les équations de Monge-Ampère, I, Manuscripta Math., 41 (1983), 1-43, MR 0786541 (86k:35044), Zbl 0579.35027; Sur les équations de MongeAmpère, Arch. Rational Mech. Anal., 89 (1985), 93-122, MR 0689131 (85d:35040) Zbl 0509.35036.

[11] J. Matero, The Bieberbach-Rademacher problem for the Monge-Ampère operator, Manuscripta Math., 91 (1996), 379-391, MR 1416719 (97i:35107), Zbl 0873.35026.

[12] R. Osserman, On the inequality $\Delta u \geq f(u)$, Pacific J. Math., 7 (1957), 1641-1647, MR 0098239 (20 \#4701), Zbl 0083.09402.

[13] P. Salani, Boundary blow-up problems for Hessian equations, Manuscripta Math., 96 (1998), 281-294, MR 1638149 (99e:35071), Zbl 0907.35052.

Received July 21, 2003. Research of the first author was supported in part by an NSF grant. Research of the second author was supported in part by the National 973-Project and the Grant for Excellent Young Scholars in 21st Century from the Education Department of China.

Department of Mathematics

UNIVERSITY OF TENNESSEE

KNOXVILle TN 37996

E-mail address: guan@math.utk.edu

Department of Mathematical Sciences

Tsinghua University

BEIJING 100084

China

E-mail address: hjian@math.tsinghua.edu.cn 